\title{
Group Coordinated Control of Networked Mobile Robots with Applications to Object Transportation
}

DOI:

10.1109/TVT.2021.3093157

\section{Document Version}

Accepted author manuscript

Link to publication record in Manchester Research Explorer

\section{Citation for published version (APA):}

Hu, J., Bhowmick, P., \& Lanzon, A. (2021). Group Coordinated Control of Networked Mobile Robots with Applications to Object Transportation. IEEE Transactions on Vehicular Technology, 70(8), 8269 - 8274. [9468402]. https://doi.org/10.1109/TVT.2021.3093157

\section{Published in:}

IEEE Transactions on Vehicular Technology

\section{Citing this paper}

Please note that where the full-text provided on Manchester Research Explorer is the Author Accepted Manuscript or Proof version this may differ from the final Published version. If citing, it is advised that you check and use the publisher's definitive version.

\section{General rights}

Copyright and moral rights for the publications made accessible in the Research Explorer are retained by the authors and/or other copyright owners and it is a condition of accessing publications that users recognise and abide by the legal requirements associated with these rights.

\section{Takedown policy}

If you believe that this document breaches copyright please refer to the University of Manchester's Takedown Procedures [http://man.ac.uk/04Y6Bo] or contact uml.scholarlycommunications@manchester.ac.uk providing relevant details, so we can investigate your claim.

\section{OPEN ACCESS}




\title{
Group Coordinated Control of Networked Mobile Robots with Applications to Object Transportation
}

\author{
Junyan Hu, Member, IEEE, Parijat Bhowmick, Member, IEEE, and Alexander Lanzon, Senior Member, IEEE
}

\begin{abstract}
Inspired by the group activities of natural swarms (e.g., a flock of birds, a colony of ants, etc.), a fleet of mobile robots can be collaboratively put into work to accomplish complex real-world tasks. Depending on the nature and complexity of a problem, a multi-robot system (MRS) may need to be decomposed into several subgroups. This paper proposes a unified group coordinated control scheme for networked MRSs having multiple targets. A 'discontinuous' cooperative control law is first developed for a networked MRS to achieve individual subformations surrounding the assigned targets. A 'continuous' cooperative control protocol is then proposed to overcome the chattering phenomenon often caused by a discontinuous control action during hardware implementation. The closed-loop stability of the overall networked MRS is guaranteed via the Lyapunov theory and boundary-layer techniques. Finally, two hardware experiments (target-enclosing and object transportation) involving real mobile robots have been carried out to demonstrate the usefulness of the proposed scheme.
\end{abstract}

Index Terms-Swarm robotics, object transportation, targetenclosing, multi-vehicle systems, group coordination.

\section{INTRODUCTION}

Distributed cooperative control of multi-robot systems (MRSs) has established its worth in both theories and practice over the past two decades [1]. Some potential applications of MRSs include swarm shepherding [2], connected vehicle platooning [3], cooperative exploration in unknown environments [4], etc. As one of the useful techniques of the cooperative control framework, the formation control of autonomous systems has received significant attention from robotics, aerospace and vehicular engineering disciplines. In [5], the formation control problem for a group of nonholonomic mobile robots was addressed considering the communication time-delays. [6] proposed an extended state observer-based distributed model predictive control approach to deal with the multi-robot formation control problem in the presence of unknown disturbances. Recently, in [7], finite-time formation control of multiple nonholonomic wheeled mobile robots with a leader-following structure was studied. However, the preceding literature relied only on the simulation results to show the effectiveness of the proposed schemes, but no experimental validation involving real robots was provided.

To verify the feasibility of cooperative control techniques in real-world scenarios, numerous experiments and testing have been done involving real mobile robots. A two-layer

This work was supported by the Engineering and Physical Sciences Research Council (EPSRC) [grant number EP/R008876/1]. All research data supporting this publication are directly available within this publication.

The authors are with the Department of Electrical and Electronic Engineering, The University of Manchester, Manchester M13 9PL, U.K. (e-mail: \{Junyan.Hu, Parijat.Bhowmick, Alexander.Lanzon\}@manchester.ac.uk) formation-containment control architecture for MRSs with linear dynamics was proposed in [8]. In [9], a distributed observer-based formation control framework was developed to track the centroid of relative formations of a MRS characterised with first-order dynamics. However, these studies considered only a single group of robots, which appears to be a limitation in real-world multi-robot applications that need to split the robot team into multiple subgroups to accomplish the given task.

In some multi-robot applications (e.g. multi-target pursuit and target-enclosing mission), a team of robots may need to be split into several subgroups (also known as 'clusters') surrounding each target, depending on the positions of the targets and the complexity of the task [10]. In such cases, the analysis and design of the coordinated control strategies become more challenging as the inter-group conflicts cause significant difficulties. [11] and [12] did pioneering research on cluster consensus control problems. However, there are still many issues to overcome regarding the design and implementation of the cluster control strategies in real-world applications, especially when the targets become dynamic.

Drawn by the limitations and challenges mentioned above, we aim to develop a Group Coordinated Control (GCC) scheme in this paper for networked multi-robot applications. The control scheme first divides the participant robots into several subgroups depending on the targets (may be dynamic). A 'continuous' coordination protocol is then proposed apart from the 'discontinuous' version, which is more common in the MRS literature, to remove the chattering effect caused by the latter. Besides, a couple of hardware experiments involving real mobile robots have been conducted to test the feasibility of the proposed GCC scheme. It is also explained that the leaderfollowing consensus-seeking problem, the formation tracking problem and the cluster control problem can all be considered as special cases of the proposed GCC framework.

\section{PROBlem FORMULATION}

Consider a MRS containing $N$ robots that includes $M$ followers and $N-M$ targets. Let $\mathscr{F}=\{1,2, \ldots, M\}$ and $\mathscr{T}=\{M+1, M+2, \ldots, N\}$ be the sets of the followers and targets respectively. All the robot are connected by a weighted and directed communication graph $\mathscr{G}=(\mathscr{V}, \mathscr{E})$ having a nonempty set of nodes $\mathscr{V}=\{1,2, \ldots, N\}$ and a set of edges $\mathscr{E} \subset \mathscr{V} \times \mathscr{V}$. For any $i, j \in\{1,2, \ldots, N\}, w_{i j}$ is defined as

$$
w_{i j}=\left\{\begin{array}{l}
0 \text { when } i=j \text { or }(j, i) \notin \mathscr{E} \\
b_{j} \text { when } j \in \mathscr{T} \text { and }(j, i) \in \mathscr{E} ; \\
a_{i j} \text { when both } i, j \in \mathscr{F} \text { and }(j, i) \in \mathscr{E}
\end{array}\right.
$$


where $b_{j}$ are known positive constants.

Suppose that there are $p(p \geq 1)$ subgroups in the MRS, and the node set $\mathscr{V}$ can be partitioned into $\left\{\mathscr{V}_{1}, \ldots, \mathscr{V}_{p}\right\}$. Let $\bar{i}$ and $\mathscr{G}_{i}$ denote the subscript of the subgroup to which follower $i$ belongs and the underlying interaction topology of the subgroup $\mathscr{V}_{\bar{i}}(\forall \bar{i} \in\{1,2, \ldots, p\})$, respectively. $n_{\bar{i}}$ and $\hat{n}_{\bar{i}}$ are the number of followers and targets in subgroup $\mathscr{V}_{i}$.

Assumption 1: For each subgroup there exists at least one target which provides the reference trajectory to the followers. Any follower in a given subgroup is assumed to be either wellinformed or uninformed. For each uninformed follower, there exists at least one well-informed follower that has a directed path to that uninformed follower.

Assumption 2: $\left\{\mathscr{V}_{1}, \mathscr{V}_{2}, \ldots, \mathscr{V}_{p}\right\}$ is an acyclic partition of the node set $\mathscr{V}$.

Then the Laplacian matrix $L$ corresponding to the graph $\mathscr{G}$ can be partitioned as $L=\left[\begin{array}{cc}L_{1} & L_{2} \\ 0 & 0\end{array}\right]$ where $L_{1} \in \mathbb{R}^{M \times M}$ and $L_{2} \in \mathbb{R}^{M \times(N-M)}$. Owing to Assumption 2, the sub-Laplacian matrix $L_{1}$ can be further partitioned [11] as

$$
L_{1}=\left[\begin{array}{cccc}
L_{11} & 0 & \cdots & 0 \\
L_{21} & L_{22} & \cdots & 0 \\
\vdots & \vdots & \ddots & \vdots \\
L_{p 1} & L_{p 2} & \cdots & L_{p p}
\end{array}\right]
$$

where $L_{\overline{i i}}$ is associated with $\mathscr{G}_{i}$, and $L_{\bar{i} \bar{j}}$ represents the interaction from subgroup $\bar{j}$ to subgroup $\bar{i}$ for any $\bar{i}, \bar{j} \in\{1, \ldots, p\}$ and $\bar{i} \neq \bar{j}$.

Assumption 3: Each sub-Laplacian matrix $L_{\bar{i} \bar{j}}$ derived from $L_{1}$ for all $\bar{j}<\bar{i}$ where $\bar{i}, \bar{j} \in\{1,2, \ldots, p\}$ is a zero-row-sum matrix.

For the $\bar{i}^{\text {th }}$ subgroup for each $\bar{i} \in\{1,2, \ldots, p\}$, the desired time-varying sub-formation is specified by the vector $\bar{h}_{\bar{i}}(t)=\left[h_{\varsigma_{i}+1}^{\top}(t), \ldots, h_{\varsigma_{\bar{i}}+n_{\bar{i}}}^{\top}(t)\right]^{\top}$ where $\varsigma_{\bar{i}}=\sum_{k=0}^{\bar{i}-1} n_{k}$ and each element in $\bar{h}_{\bar{i}}(t)$ is assumed to be piecewise continuously differentiable. It is easy to verify that $h_{F}(t)=\left[\bar{h}_{1}^{\top}(t), \ldots, \bar{h}_{p}^{\top}(t)\right]^{\top}=$ $\left[h_{1}^{\top}(t), \ldots, h_{M}^{\top}(t)\right]^{\top}$ gives the formation vector for the entire MRS. $\bar{x}_{\bar{i}}=\left[x_{\varsigma_{\bar{i}} \pm 1}^{\top}(t), \ldots, x_{\varsigma_{\bar{i}}+n_{\bar{i}}}^{\top}(t)\right]^{\top}$ and $\hat{x}_{\bar{i}}=$ $\left[x_{\hat{\varsigma}_{\bar{i}}+1}^{\top}(t), \ldots, x_{\hat{\varsigma}_{\bar{i}}+\hat{n}_{\bar{i}}}^{\top}(t)\right]^{\top}$ represent the state vector of the followers and the targets respectively of the $\bar{i}^{\text {th }}$ subgroup, where $\hat{\varsigma}_{\bar{i}}=M+\sum_{k=0}^{\bar{i}-1} \hat{n}_{k}$.

The MRS is said to achieve group formation tracking with multiple targets if for any given set of bounded initial states,

$$
\lim _{t \rightarrow \infty}\left(\bar{x}_{\bar{i}}(t)-\bar{h}_{\bar{i}}(t)-\mathbf{1}_{n_{\bar{i}}} \sum_{k=\hat{\varsigma}_{\bar{i}}+1}^{\hat{\varsigma}_{\bar{i}+\hat{n}_{\bar{i}}}} \alpha_{k} x_{k}(t)\right)=0
$$

$\forall \bar{i} \in\{1,2, \ldots, p\}$ where $\alpha_{k}$ denote the positive constants that satisfy the constraint $\sum_{k=\hat{\varsigma}_{\bar{i}}+1}^{\hat{\varsigma}_{\bar{i}} \hat{n}_{\bar{i}}} \alpha_{k}=1$.

This article mainly solves the following four problems: (i) under what conditions the group coordination tasks can be accomplished; (ii) how to construct the control protocol to form the desired sub-formations around the targets; (iii) how to design continuous control action to avoid chattering effect during hardware implementation; (iv) how to implement the proposed method in real-time hardware experiments.

\section{MAIN RESULTS}

\section{A. Linearization of the nonlinear robot dynamics}

It is assumed that each mobile robot has the same mechanical structure and they are described by the following set of dynamic equations involving the nonholonomic constraints

$$
\Sigma_{R}:\left\{\begin{array}{l}
\dot{p}_{x i}=v_{i} \cos \theta_{i}, \dot{p}_{y i}=v_{i} \sin \theta_{i}, \dot{\theta}_{i}=\omega_{i} \\
\dot{v}=f_{i} / m_{i}, \dot{\omega}=\tau_{i} / J_{i}
\end{array}\right.
$$

where $p_{x i}$ and $p_{y i}$ represent the position of the mass centre of the $i^{\text {th }}$ robot, $\theta_{i}$ is the orientation, $v_{i}$ and $\omega_{i}$ are respectively the linear and angular velocities, $m_{i}$ is the mass, $J_{i}$ is the mass moment of inertia, $\tau_{i}$ is the torque applied to the robot and $f_{i}$ is the force generated due to applying the torque $\tau_{i}$.

The X-axis and Y-axis components of the head position of the robot can be expressed by

$$
\tilde{p}_{x i}=p_{x i}+l \cos \theta_{i}, \tilde{p}_{y i}=p_{y i}+l \sin \theta_{i}
$$

where $l$ is the distance between these two points. Now, being motivated by [13], we apply the following inverse coordinate transformation on the nonlinear dynamics $\Sigma_{R}$ of the robot (4)

$$
\begin{aligned}
{\left[\begin{array}{l}
f_{i} \\
\tau_{i}
\end{array}\right]=} & {\left[\begin{array}{cc}
\frac{1}{m_{i}} \cos \left(\theta_{i}\right) & -\frac{l}{J_{i}} \sin \left(\theta_{i}\right) \\
\frac{1}{m_{i}} \sin \left(\theta_{i}\right) & \frac{l}{J_{i}} \cos \left(\theta_{i}\right)
\end{array}\right]^{-1} \times } \\
& {\left[\begin{array}{l}
u_{x i}+v_{i} \omega_{i} \sin \left(\theta_{i}\right)+l \omega_{i}^{2} \cos \left(\theta_{i}\right) \\
u_{y i}+v_{i} \omega_{i} \cos \left(\theta_{i}\right)+l \omega_{i}^{2} \cos \left(\theta_{i}\right)
\end{array}\right] \quad \forall i \in\{1,2, \ldots, N\} }
\end{aligned}
$$

and obtain a fourth-order linearized dynamics

$$
\dot{x}_{i}=A x_{i}+B u_{i} \quad \forall i \in\{1,2, \ldots, N\}
$$

where the matrices $A=\left[\begin{array}{llll}0 & 1 & 0 & 0 \\ 0 & 0 & 0 & 0 \\ 0 & 0 & 0 & 1 \\ 0 & 0 & 0 & 0\end{array}\right], B=\left[\begin{array}{ll}0 & 0 \\ 1 & 0 \\ 0 & 0 \\ 0 & 1\end{array}\right], x_{i}=$ $\left[\begin{array}{llll}\tilde{p}_{x i}^{\top} & v_{x i}^{\top} & \tilde{p}_{y i}^{\top} & v_{y i}^{\top}\end{array}\right]^{\top}$ and $u_{i}=\left[\begin{array}{ll}u_{x i}^{\top} & u_{y i}^{\top}\end{array}\right]^{\top}$. It can be readily verified that the pair $(A, B)$ is always stabilizable and $B$ has full column-rank. Hence, there exists a matrix $\bar{B}$ depending on the left null space of $B$ such that $\bar{B} B=0$.

Assumption 4: Let $u_{k}(t)$ for all $k \in \mathscr{T}$ represent the exogenous input applied to the $k^{\text {th }}$ target which is independent of all other robots and the network topology. We assume $\left\|u_{k}(t)\right\| \leq \sigma$ for all $t \geq 0$ for a given $\sigma \in \mathbb{R}_{>0}$.

\section{B. Group coordinated control protocol design}

We define the local group coordinated tracking error $\xi_{i}$ of the $i^{\text {th }}$ follower robot for all $i \in \mathscr{F}$ with respect to the neighboring robots as $\xi_{i}=\sum_{j=1}^{M} w_{i j}\left(\left(x_{i}-h_{i}\right)-\left(x_{j}-h_{j}\right)\right)+$ $\sum_{k=M+1}^{N} w_{i k}\left(\left(x_{i}-h_{i}\right)-x_{k}\right)$.

The following theorem proposes a distributed control law for a MRS to achieve sub-formations by each individual subgroups along with keep tracking the respective targets.

Theorem 1: If the Assumptions 1-4 hold and the desired formation configuration $h_{i} \in \mathbb{R}^{n}$ be chosen such that

$$
\bar{B} A h_{i}-\bar{B} \dot{h}_{i}=0 \quad \forall t \geq 0 .
$$

Then the MRS achieves the group formation tracking objectives by the following distributed control law

$$
u_{i}=c K \xi_{i}+\gamma_{i}-\mu f\left(\xi_{i}\right) \quad \forall i \in \mathscr{F}
$$


where $c>0 ; \mu>\sigma$ for a given $\sigma \geq 0 ; \gamma_{i}=\tilde{B} \dot{h}_{i}-\tilde{B} A h_{i}$; $K=-R^{-1} B^{\top} P$ where $P=P^{\top}>0$ be the unique solution to the algebraic Riccati equation (ARE)

$$
P A+A^{\top} P-P B R^{-1} B^{\top} P+Q=0
$$

for given $Q>0$ and $R>0$ and the nonlinear smooth function $f\left(\xi_{i}\right)$ is defined for all $i \in \mathscr{F}$ as

$$
f\left(\xi_{i}\right)=\left\{\begin{array}{cc}
\frac{B^{\top} P \xi_{i}}{\left\|B^{\top} P \xi_{i}\right\|} & \text { when }\left\|B^{\top} P \xi_{i}\right\| \neq 0 \\
0 & \text { when }\left\|B^{\top} P \xi_{i}\right\|=0
\end{array} \quad \forall t \geq 0 .\right.
$$

Proof: In this proof, we will use the following notations: $x_{F}=\left[x_{1}^{\top}, \ldots, x_{M}^{\top}\right]^{\top}, x_{E}=\left[x_{M+1}^{\top}, \ldots, x_{N}^{\top}\right]^{\top}$, $u_{E}=\left[u_{M+1}^{\top}, \ldots, u_{N}^{\top}\right]^{\top}, \gamma=\left[\gamma_{1}^{\top}, \gamma_{2}^{\top}, \ldots, \gamma_{M}^{\top}\right]^{\top}$ and $F(\xi)=\left[f^{\top}\left(\xi_{1}\right), f^{\top}\left(\xi_{2}\right), \ldots, f^{\top}\left(\xi_{M}\right)\right]^{\top}$.

Let the local formation tracking error of each follower be defined as $z_{i}=x_{i}-h_{i} \forall i \in \mathscr{F}$ and $z_{F}=\left[z_{1}^{\top}, \ldots, z_{M}^{\top}\right]^{\top}$. Then $\xi_{F}=\left[\xi_{1}^{\top}, \ldots, \xi_{M}^{\top}\right]^{\top}$ can be expressed in the Kronecker product form as

$$
\xi_{F}=\left(L_{1} \otimes I_{n}\right) z_{F}+\left(L_{2} \otimes I_{n}\right) x_{E} .
$$

Applying the proposed distributed control law, the expression for $\dot{\xi}_{F}$ is given by

$$
\begin{aligned}
\dot{\xi}_{F}= & \left(I_{M} \otimes A+c L_{1} \otimes B K\right) \xi_{F}+\left(L_{1} \otimes A\right) h_{F} \\
& -\left(L_{1} \otimes I_{n}\right) \dot{h}_{F}+\left(L_{1} \otimes B\right) \gamma+\left(L_{2} \otimes B\right) u_{E} \\
& -\mu\left(L_{1} \otimes B\right) F(\xi) .
\end{aligned}
$$

We will now establish the asymptotic stability of the formation tracking error dynamics derived in (12) by applying the Lyapunov stability approach. Consider the following Lyapunov function candidate

$$
V_{1}=\xi_{F}^{\top}(\Delta \Xi \otimes P) \xi_{F},
$$

where $\Delta \Xi=\operatorname{diag}\left\{\varphi_{1}, \ldots, \varphi_{M}\right\}$ with $\varphi_{i}>0 \forall i \in \mathscr{F}$. Utilising the formation feasibility condition (7), now the time derivative of $V_{1}$ is computed along any trajectory of (12)

$$
\begin{aligned}
\dot{V}_{1}= & \xi_{F}^{\top}\left[\Delta \Xi \otimes\left(P A+A^{\top} P\right)-c\left(\Delta \Xi L_{1}+L_{1}^{\top} \Delta \Xi\right) \otimes \Gamma\right] \xi_{F} \\
& +2 \xi_{F}^{\top}\left(\Delta \Xi L_{2} \otimes P B\right) u_{E}-2 \mu \xi_{F}^{\top}\left(\Delta \Xi L_{1} \otimes P B\right) F(\xi)
\end{aligned}
$$

denoting $\Gamma=P B R^{-1} B^{\top} P$.

By Cauchy-Schwarz inequality, the term $2 \mu \xi_{F}^{\top}\left(\Delta \Xi L_{1} \otimes\right.$ $P B) F(\xi)$ can be simplified as following

$$
\begin{aligned}
& -2 \mu \xi_{F}^{\top}\left(\Delta \Xi L_{1} \otimes P B\right) F(\xi) \\
\leq & -2 \mu \sum_{i=1}^{M} \varphi_{i} \sum_{k=M+1}^{N} w_{i k}\left\|B^{\top} P \xi_{i}\right\|
\end{aligned}
$$

where $\varphi_{i}$ is the $i^{\text {th }}$ diagonal element of $\Delta \Xi$. Subsequently, the term $2 \xi_{F}^{\top}\left(\Delta \Xi L_{2} \otimes P B\right) u_{E}$ can also be simplified as

$$
\begin{aligned}
& 2 \xi_{F}^{\top}\left(\Delta \Xi L_{2} \otimes P B\right) u_{E}=2 \sum_{i=1}^{M} \varphi_{i} \sum_{k=M+1}^{N} w_{i k} \xi_{i}^{\top} P B u_{k} \\
\leq & 2 \sigma \sum_{i=1}^{M} \varphi_{i} \sum_{k=M+1}^{N} w_{i k}\left\|B^{\top} P \xi_{i}\right\|
\end{aligned}
$$

via exploiting Assumption 4. Now substituting (15) and (16) into (14), we find

$$
\begin{gathered}
\dot{V}_{1} \leq \xi_{F}^{\top}\left[\Delta \Xi \otimes\left(P A+A^{\top} P\right)-c\left(\Delta \Xi L_{1}+L_{1}^{\top} \Delta \Xi\right) \otimes \Gamma\right] \xi_{F} \\
-2(\mu-\sigma) \sum_{i=1}^{M} \varphi_{i} \sum_{k=M+1}^{N} w_{i k}\left\|B^{\top} P \xi_{i}\right\| .
\end{gathered}
$$

Upon choosing $c \geq \frac{\lambda_{\max }(\Delta \Xi)}{\lambda_{\min }\left(\Delta \Xi L_{1}+L_{1}^{\top} \Delta \Xi\right)}$ and $\mu>\sigma$, the expression of $\dot{V}_{1}$ reduces to

$$
\dot{V}_{1} \leq \xi_{F}^{\top}\left(\Delta \Xi \otimes\left(P A+A^{\top} P-\Gamma\right)\right) \xi_{F} .
$$

This implies $\dot{V}_{1}<0$ since $\left(P A+A^{\top} P-\Gamma\right)=-Q<0$ via (9) and furthermore, $\dot{V}_{1}=0$ when $\xi_{F}=0$. Therefore, the group coordinated tracking error dynamics $\dot{\xi}_{F}$ is asymptotically stable and hence, we have

$$
\lim _{t \rightarrow \infty}\left(x_{F}-h_{F}-\left(-L_{1}^{-1} L_{2} \otimes I_{n}\right) x_{E}\right)=0
$$

Now the expression (19) can be rearranged as

$$
\lim _{t \rightarrow \infty}\left(\left[\begin{array}{c}
\bar{x}_{1}-\bar{h}_{1}-\left(e_{1} \otimes I_{n}\right) \hat{x}_{1} \\
\vdots \\
\bar{x}_{p}-\bar{h}_{p}-\left(e_{p} \otimes I_{n}\right) \hat{x}_{p}
\end{array}\right]\right)=0
$$

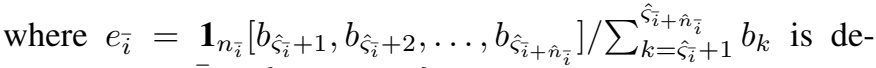
noted for all $\bar{i} \in\{1,2, \ldots, p\}$. The above expression proves that the predefined sub-formation is achieved by all the subgroups and the follower robots in each subgroup keep tracking the convex combination of the states of the targets assigned to the respective subgroup. This completes the proof.

\section{Group coordinated control with continuous control action}

An inherent drawback of the discontinuous GCC protocol (8) is that it causes unavoidable 'chattering effect' in the control signal during hardware implementation because of the imperfections of the switching devices [14]. One possible approach to minimize the chattering problem is to apply the 'boundary layer technique' [15] to obtain a legitimate continuous approximation of the discontinuous nonlinear function $f\left(\xi_{i}\right)$ used in (8). Exploiting this idea, we modify the discontinuous GCC protocol as

$$
u_{i}=c K \xi_{i}+\gamma_{i}-\mu f\left(\xi_{i}\right) \quad \forall i \in \mathscr{F}
$$

where $f\left(\xi_{i}\right)$ is defined as

$$
f\left(\xi_{i}\right)= \begin{cases}\frac{B^{\top} P \xi_{i}}{\left\|B^{\top} P \xi_{i}\right\|} & \text { when }\left\|B^{\top} P \xi_{i}\right\|>\kappa, \\ \frac{B^{\top} P \xi_{i}}{\kappa} & \text { when }\left\|B^{\top} P \xi_{i}\right\| \leq \kappa\end{cases}
$$

for a pre-specified $\kappa>0$, while the remaining control parameters of (21) are the same as declared in Theorem 1. The constant parameter $\kappa$ specifies a lower bound of the 2-norm of the weighted tracking error $B^{\top} P \xi_{i}$. Although in this method, the formation tracking error cannot be made exactly zero but can be small enough to achieve satisfactory performance.

The next theorem formally described the continuous version of the GCC protocol developed for a MRS to achieve the group formation tracking objectives. 
Theorem 2: Let the Assumptions 1-4 hold. Suppose that the formation feasibility constraint $\bar{B} A h_{i}-\bar{B} \dot{h}_{i}=0$ holds for the desired $h_{i}$ for all $i \in \mathscr{F}$. Define $\beta=$ $\frac{-\lambda_{\max }\left(P A+A^{\top} P-P B R^{-1} B^{\top} P\right)}{\lambda_{\max }(P)}$ where $P=P^{\top}>0$ is the unique solution to the ARE (9) for given $Q>0$ and $R>0$. Then the MRS precisely achieves the GCC objectives under the application of the distributed control protocol (21) ensuring the global group formation tracking error $\xi_{F}(t)$ to remain within the bounded set

$$
\mathscr{D}=\left\{\xi_{F}(t):\left\|\xi_{F}(t)\right\|^{2} \leq \frac{2 \sigma \kappa \sum_{i=1}^{M} \varphi_{i} \sum_{k=M+1}^{N} w_{i k}}{\beta \lambda_{\min }(\Delta \Xi) \lambda_{\min }(P)}\right\}
$$

for all $t \geq 0$.

Proof: The proof proceeds in the similar direction as of Theorem 1. The group formation tracking error dynamics of the robots $\left(\dot{\xi}_{F}\right)$ is given by.

$$
\begin{aligned}
\dot{\xi}_{F}= & \left(I_{M} \otimes A+c L_{1} \otimes B K\right) \xi_{F}+\left(L_{2} \otimes B\right) u_{E} \\
& -\mu\left(L_{1} \otimes B\right) F(\xi)
\end{aligned}
$$

Consider the Lyapunov function candidate $V_{2}\left(\xi_{F}\right)=$ $\xi_{F}^{\top}(\Delta \Xi \otimes P) \xi_{F}$ similar to Theorem 1. It is easy to verify $V_{2}\left(\xi_{F}\right)>0$ and $V_{2}\left(\xi_{F}\right)=0$ only when $\xi_{F}=0$. Then $\dot{V}_{2}\left(\xi_{F}\right)$ is derived along the trajectories of (24)

$$
\begin{aligned}
\dot{V}_{2}\left(\xi_{F}\right)= & \xi_{F}^{\top}\left(\Delta \Xi \otimes\left(P A+A^{\top} P-\Gamma\right)\right) \xi_{F}+2 \xi_{F}^{\top}\left(\Delta \Xi L_{2}\right. \\
& \otimes P B) u_{E}-2 \mu \xi_{F}^{\top}\left(\Delta \Xi L_{1} \otimes P B\right) F(\xi) .
\end{aligned}
$$

While simplifying the terms $\xi_{F}^{\top}\left(\Delta \Xi L_{2} \otimes P B\right) u_{E}$ and $2 \mu \xi_{F}^{\top}\left(\Delta \Xi L_{1} \otimes P B\right) F(\xi)$, we will eventually encounter the norm $\left\|B^{\top} P \xi_{i}(t)\right\|$ as found in both (15) and (16). In this theorem, due to dealing with the nonlinear function $f\left(\xi_{i}\right)$ given in (22), the following three cases would appear: Case I. $\left\|B^{\top} P \xi_{i}(t)\right\|>\kappa \forall i \in \mathscr{F}$; Case II. $\left\|B^{\top} P \xi_{i}(t)\right\| \leq \kappa \forall i \in$ $\mathscr{F}$; and Case III. other than Cases I and II.

Case I. When $\left\|B^{\top} P \xi_{i}(t)\right\|>\kappa \forall i \in \mathscr{F}$, it follows directly from (15) that

$$
\begin{aligned}
-2 \mu \xi_{F}^{\top}\left(\Delta \Xi L_{1} \otimes P B\right) F(\xi) & \\
& \leq-2 \mu \sum_{i=1}^{M} \varphi_{i} \sum_{k=M+1}^{N} w_{i k}\left\|B^{\top} P \xi_{i}\right\|
\end{aligned}
$$

$\forall i \in \mathscr{F}$ and finally, we will end up with

$$
\dot{V}_{2}\left(\xi_{F}\right) \leq \xi_{F}^{\top}\left[\Delta \Xi \otimes\left(P A+A^{\top} P-\Gamma\right)\right] \xi_{F}
$$

by choosing $c \geq \frac{\lambda_{\max }(\Delta \Xi)}{\lambda_{\min }\left(\Delta \Xi L_{1}+L_{1}^{\top} \Delta \Xi\right)}$ and $\mu>\sigma$.

Case II. When $\left\|B^{\top} P \xi_{i}(t)\right\| \leq \kappa \forall i \in \mathscr{F}$, following (15) we derive

$$
\begin{aligned}
&-2 \mu \xi_{F}^{\top}\left(\Delta \Xi L_{1} \otimes P B\right) F(\xi) \\
& \leq-2 \frac{\mu}{\kappa} \sum_{i=1}^{M} \varphi_{i} \sum_{k=M+1}^{N} w_{i k}\left\|B^{\top} P \xi_{i}\right\|^{2} \leq 0
\end{aligned}
$$

by utilizing (22) and furthermore from (16), we obtain

$$
2 \xi_{F}^{\top}\left(\Delta \Xi L_{2} \otimes P B\right) u_{E} \leq(2 \sigma \kappa) \sum_{i=1}^{M} \varphi_{i} \sum_{k=M+1}^{N} w_{i k} .
$$

Applying aforementioned simplified results (28) and (29) into (25), we have

$$
\begin{aligned}
\dot{V}_{2}\left(\xi_{F}\right) \leq & \xi_{F}^{\top}\left[\Delta \Xi \otimes\left(P A+A^{\top} P-\Gamma\right)\right] \xi_{F} \\
& +(2 \sigma \kappa) \sum_{i=1}^{M} \varphi_{i} \sum_{k=M+1}^{N} w_{i k} .
\end{aligned}
$$

Case III. Here, we deal with those cases where $\left\|B^{\top} P \xi_{i}(t)\right\|$ does not satisfy either of Cases I and II. Without loss of generality, we assume that $\left\|B^{\top} P \xi_{i}(t)\right\|>\kappa$ for $i \in\{1,2, \ldots, q\}$ where $2 \leq q \leq M-1$ and $\left\|B^{\top} P \xi_{i}(t)\right\| \leq \kappa$ for $i \in$ $\{q, q+1, \ldots, M\}$. Now following again (26), we have

$$
\begin{aligned}
-2 \mu \xi_{F}^{\top}\left(\Delta \Xi L_{1} \otimes P B\right) F(\xi) & \\
& \leq-2 \mu \sum_{i=1}^{q} \varphi_{i} \sum_{k=M+1}^{N} w_{i k}\left\|B^{\top} P \xi_{i}\right\|
\end{aligned}
$$

$\forall i \in\{1,2, \ldots, q\}$ and via following (29), we derive

$$
\begin{aligned}
2 \xi_{F}^{\top}\left(\Delta \Xi L_{2} \otimes P B\right) u_{E} \leq & 2 \sigma \sum_{i=1}^{q} \varphi_{i} \sum_{\substack{k=M+1 \\
M-q}}^{N} w_{i k}\left\|B^{\top} P \xi_{i}\right\| \\
& +(2 \sigma \kappa) \sum_{i=1}^{N} \varphi_{i} \sum_{k=M+1}^{N} w_{i k} .
\end{aligned}
$$

Applying the results (31) and (32) back into (17), we obtain

$$
\begin{aligned}
\dot{V}_{2}\left(\xi_{F}\right) \leq & \xi_{F}^{\top}\left[\Delta \Xi \otimes\left(P A+A^{\top} P-\Gamma\right)\right] \xi_{F} \\
& +2 \sigma \kappa \sum_{i=1}^{M-q} \varphi_{i} \sum_{k=M+1}^{N} w_{i k} .
\end{aligned}
$$

After analysing all three cases (Cases I, II and III) presented above, it can be inferred that if $\dot{V}_{2}\left(\xi_{F}\right)$ satisfies (30), then it will satisfy both (27) and (33). Hence, in order to guarantee asymptotic stability of the error dynamics $\dot{\xi}_{F}$ in all three cases, it needs to be established that (30) implies uniform boundedness of the Lyapunov function $V_{2}\left(\xi_{F}\right)$ and exponential convergence of the group formation tracking error $\xi_{F}$.

The expression (30) can be rewritten as

$$
\begin{aligned}
\dot{V}_{2}\left(\xi_{F}\right) \leq & -\beta V_{2}\left(\xi_{F}\right)+2 \sigma \kappa \sum_{i=1}^{M} \varphi_{i} \sum_{k=M+1}^{N} w_{i k} \\
& +\xi_{F}^{\top}\left[\Delta \Xi \otimes\left(P A+A^{\top} P+\beta P-\Gamma\right)\right] \xi_{F} .
\end{aligned}
$$

Since $\beta=\frac{-\lambda_{\max }\left(P A+A^{\top} P-\Gamma\right)}{\lambda_{\max }(P)}>0$, it follows from (34) that

$$
\dot{V}_{2}\left(\xi_{F}\right) \leq-\beta V_{2}\left(\xi_{F}\right)+2 \sigma \kappa \sum_{i=1}^{M} \varphi_{i} \sum_{k=M+1}^{N} w_{i k} .
$$

Now by applying the Comparison Lemma, we can find a solution (in terms of inequality) of $V_{2}\left(\xi_{F}\right)$ from the linear differential inequality (35) as

$$
\begin{gathered}
V_{2}\left(\xi_{F}\right) \leq\left(V_{2}\left(\xi_{F}(0)\right)-\left(\frac{2}{\beta} \sigma \kappa\right) \sum_{i=1}^{M} \varphi_{i} \sum_{k=M+1}^{N} w_{i k}\right) e^{-\beta t} \\
+\left(\frac{2}{\beta} \sigma \kappa\right) \sum_{i=1}^{M} \varphi_{i} \sum_{k=M+1}^{N} w_{i k}
\end{gathered}
$$


This implies that the function $V_{2}\left(\xi_{F}\right)$ exponentially converges to the set $\left\{V_{2}\left(\xi_{F}\right): V_{2}\left(\xi_{F}\right) \leq\left(\frac{2}{\beta} \sigma \kappa\right) \sum_{i=1}^{M} \varphi_{i} \sum_{k=M+1}^{N} w_{i k}\right\}$ for any bounded initial condition $\xi_{F}(0) \in \mathbb{R}^{n M}$ with a convergence rate not lesser than $e^{-\beta t}$. This confirms that $V_{2}\left(\xi_{F}\right)$ remains uniformly bounded over all $t \geq 0$. We may also find a lower bound of $V_{2}\left(\xi_{F}\right)=\xi_{F}^{\top}(\Delta \Xi \otimes P) \xi_{F}$ as $V_{2}\left(\xi_{F}\right) \geq \lambda_{\min }(\Delta \Xi) \lambda_{\min }(P)\left\|\xi_{F}\right\|^{2}$ by utilizing the property $\lambda_{\min }[M] x^{\top} x \leq x^{\top} M x \leq \lambda_{\max }[M] x^{\top} x$ for any symmetric positive definite matrix $M$. By exploiting the upper and lower bounds of $V_{2}\left(\xi_{F}\right)$, it can be ascertained that the group formation tracking error $\dot{\xi}_{F}$ of the follower robots exponentially converges to the set $\mathscr{D}$, as defined in (23), with a convergence rate not lesser than $e^{-\beta t}$. The same conclusion can be drawn for Case I and Case III as well. This completes the proof.

Remark 1: By choosing $\hat{n}_{\bar{i}}=1 \forall \bar{i} \in\{1,2, \ldots, p\}$, the proposed GCC protocol becomes suitable for dealing with the cluster control problems, as discussed in [12]. Moreover, when specialised to $p=1$ and $M=N-1$, the GCC scheme captures the common formation control problem [16]. Furthermore, the GCC problem resembles the leader-following consensus-seeking (or rendezvous) problems such as [17] when $h_{i}=0 \forall i \in \mathscr{F}, p=1$ and $M=N-1$.

Remark 2: It is important to implement a reliable collisionavoidance strategy to ensure a collision-free movement of the robots while operating in a cluttered environment. In real applications, each robot can be coordinated by a primary controller (i.e. the GCC protocol) and a secondary controller, facilitating collision avoidance. The idea is to re-route the robots' navigation paths upon encountering the obstacles by generating repulsive forces based on the locations of the obstacles. The formation configuration can be recovered once the obstacles are bypassed since the robots are still connected via the network.

\section{EXPERIMENTAL VALIDATION}

In this section, two lab-based hardware experiments have been conducted to validate the proposed GCC scheme on small-scale mobile robots [18]. As shown in Fig. 1, the experimental platform includes a rectangular arena and a digital camera connected to a laptop that operates the camera tracking system [19]. The state information is transmitted to the computer via the ROS communication framework, and then the relative state information is sent to the corresponding robot using an RF transceiver module. Since the control law relies only on the local information, the present experimental setup is suitable for validating the feasibility of the proposed scheme. However, in more complex and challenging environments, advanced robots equipped with onboard sensors to detect the relative position and bearing information would be more appropriate. In that scenario, the camera tracking system and the base station will no longer be required. The video of the experimental results can be found in the Supplementary Material.

\section{A. Experiment 1: Multi-target enclosing}

Experiment 1 involves six networked mobile robots to perform the multi-target enclosing task. Three static targets

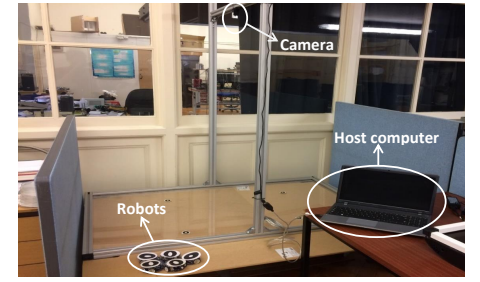

Fig. 1. The experimental arena includes the overhead camera tracking system, the base station (i.e. the host PC) and the small-scale mobile robots.

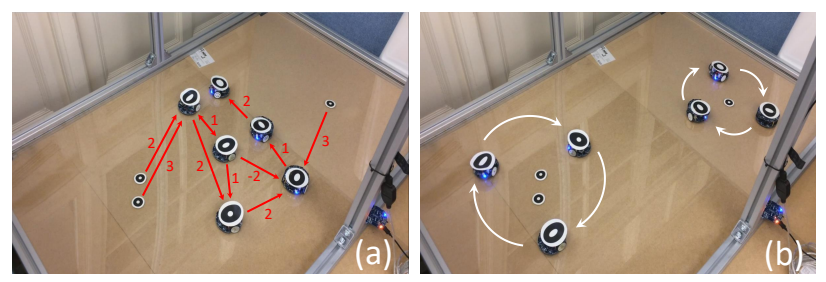

Fig. 2. Position of the networked mobile robots at the time instants: (a) $t=0$ $\mathrm{s}$ and (b) $t=60 \mathrm{~s}$.

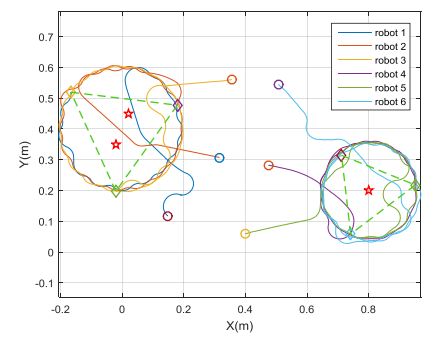

(a)

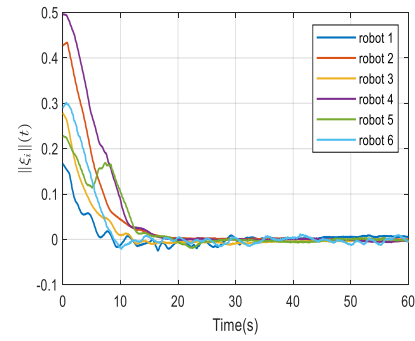

(b)
Fig. 3. (a) Position trajectories of all six robots in the $\mathrm{X}-\mathrm{Y}$ plane during the course of achieving multi-target enclosing mission. The symbols $\circ$ and $\diamond$ indicate respectively the initial and final positions of a robot while the red stars indicate the locations of targets. (b) Time-variation of the 2-norm of the group formation tracking error of the robots.

are considered in this experiment. The robots are clustered into two subgroups each containing three robots depending on the relative locations of the given targets on the arena. Fig. 2(a) describes the directed communication topology among all six robots and the positions of three given targets (marked by Black annular disc).

The robots are divided into two subgroups surrounding the given targets. Subgroup 1 has two targets to track while Subgroup 2 has a single target. The progress during the hardware experiment on multi-target enclosing is manifested by a few real-time snapshots shown in Fig. 2(a)-2(b) and for better understanding, the position trajectories of the robots during the experiment are also plotted in the X-Y plane (see Fig. 3(a)). It can be seen that both the subgroups have formed circular time-varying sub-formations around the given targets, that is, the targets have been enclosed by all the robots. Timevariation of the 2-norm of the group formation tracking error $\xi_{i}$ of all six robots are shown in Fig. 3(b) from which it is clear that both the subgroups precisely achieve the individual subformations surrounding the targets. Hence, it can be concluded that the multi-target enclosing mission has been successfully accomplished under the proposed GCC scheme. 

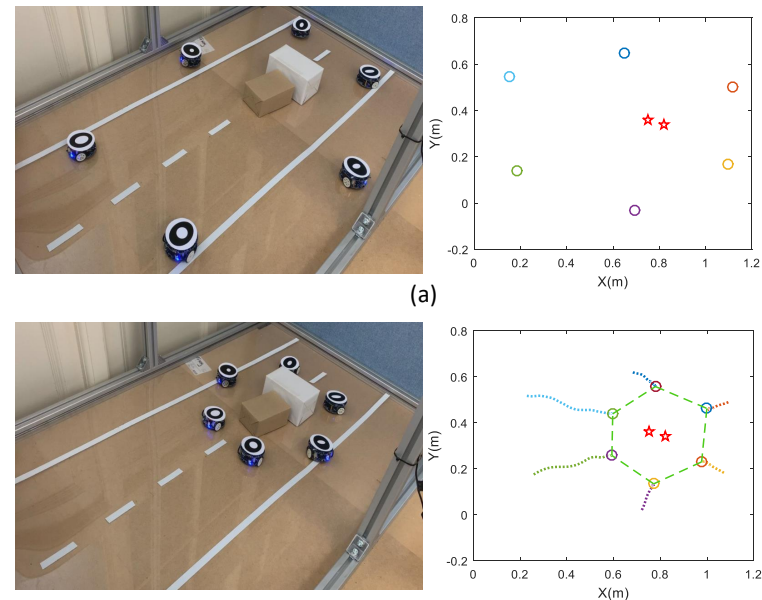

(b)
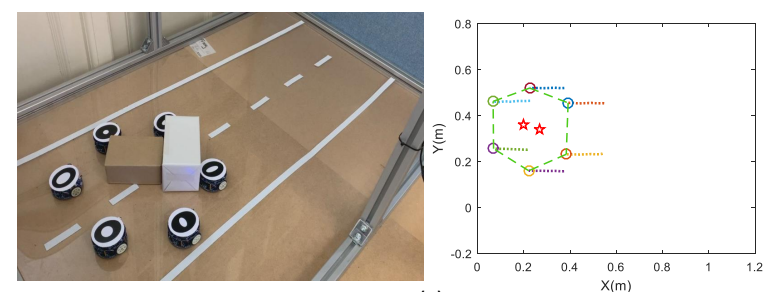

(c)

Fig. 4. (a) Initial orientation of the robots and location of the objects; (b) All six robots have achieved a hexagonal formation and thereby formed a cage surrounding the objects; (c) The whole cage along with the objects reaches the other end of the arena - mission accomplished.

\section{B. Experiment 2: Cooperative object transportation}

The strategy of group formation tracking and control has been exploited here to perform the task of object transportation by a MRS. Instead of physical targets, in this approach, the objects to be transported are considered as virtual targets. The virtual targets are made to move in a direction that leads to reaching the destination where the objects needs to be carried. By the GCC principle, the assembly of robots (i.e. the whole formation) form a cage-like structure surrounding the objects so that when the cage moves, the objects also moves being physically pushed by the robots.

In the experiment, six mobile robots are engaged in transporting two light-weight boxes from one end of the arena to the other end in a cooperative manner. The experimental observation is shown in Fig. 4(a)-4(c). Each figure contains also a X-Y graph which shows the position trajectories of the robots and the virtual targets (marked by the red star). To achieve the goal, the assembly of robots (i.e. the whole formation) makes a cage-like structure surrounding the objects and then pushes it towards the desired location. From all these figures, it can be concluded that the object transportation mission can be accomplished under the application of the proposed distributed GCC strategy.

\section{CONCLUSION}

This paper proposes a group coordinated control scheme for MRSs connected via directed communication topology. Given a cooperative control task, a MRS can be divided into several subgroups and can be driven to attain the desired subformations around the corresponding targets. To achieve this goal, both 'discontinuous' and 'continuous' cooperative control protocols have been proposed. Two lab-based experiments involving wheeled mobile robots were conducted to test the feasibility and usefulness of the proposed framework.

\section{REFERENCES}

[1] Z. Li and Z. Duan, Cooperative control of multi-agent systems: a consensus region approach. CRC Press, 2014.

[2] J. Hu, A. E. Turgut, T. Krajník, B. Lennox, and F. Arvin, "Occlusionbased coordination protocol design for autonomous robotic shepherding tasks," IEEE Transactions on Cognitive and Developmental Systems, (in press), 2020.

[3] D. Li and G. Guo, "Prescribed performance concurrent control of connected vehicles with nonlinear third-order dynamics," IEEE Transactions on Vehicular Technology, vol. 69, no. 12, pp. 14 793-14 802, 2020.

[4] J. Hu, H. Niu, J. Carrasco, B. Lennox, and F. Arvin, "Voronoi-based multi-robot autonomous exploration in unknown environments via deep reinforcement learning," IEEE Transactions on Vehicular Technology, vol. 69, no. 12, pp. 14413-14423, 2020.

[5] R. Cepeda-Gomez and L. F. Perico, "Formation control of nonholonomic vehicles under time delayed communications," IEEE Transactions on Automation Science and Engineering, vol. 12, no. 3, pp. 819-826, 2015.

[6] A. Liu, W.-A. Zhang, L. Yu, H. Yan, and R. Zhang, "Formation control of multiple mobile robots incorporating an extended state observer and distributed model predictive approach," IEEE Transactions on Systems, Man, and Cybernetics: Systems, vol. 50, no. 11, pp. 4587-4597, 2018.

[7] Y. Cheng, R. Jia, H. Du, G. Wen, and W. Zhu, "Robust finite-time consensus formation control for multiple nonholonomic wheeled mobile robots via output feedback," International Journal of Robust and Nonlinear Control, vol. 28, no. 6, pp. 2082-2096, 2018.

[8] J. Hu, P. Bhowmick, and A. Lanzon, "Two-layer distributed formationcontainment control strategy for linear swarm systems: Algorithm and experiments," International Journal of Robust and Nonlinear Control, vol. 30, no. 16, pp. 6433-6453, 2020.

[9] G. Antonelli, F. Arrichiello, F. Caccavale, and A. Marino, "Decentralized time-varying formation control for multi-robot systems," The International Journal of Robotics Research, vol. 33, no. 7, pp. 1029-1043, 2014.

[10] J. Hu, P. Bhowmick, I. Jang, F. Arvin, and A. Lanzon, "A decentralized cluster formation containment framework for multi-robot systems," IEEE Transactions on Robotics, (in press), 2021.

[11] J. Qin and C. Yu, "Cluster consensus control of generic linear multiagent systems under directed topology with acyclic partition," Automatica, vol. 49, no. 9, pp. 2898-2905, 2013.

[12] W. Xia and M. Cao, "Clustering in diffusively coupled networks," Automatica, vol. 47, no. 11, pp. 2395-2405, 2011.

[13] W. Ren and E. Atkins, "Distributed multi-vehicle coordinated control via local information exchange," International Journal of Robust and Nonlinear Control, vol. 17, no. 10-11, pp. 1002-1033, 2007.

[14] J. Hu, P. Bhowmick, and A. Lanzon, "Distributed adaptive time-varying group formation tracking for multiagent systems with multiple leaders on directed graphs," IEEE Transactions on Control of Network Systems, vol. 7, no. 1, pp. 140-150, 2020.

[15] Z. Li, Z. Duan, W. Ren, and G. Feng, "Containment control of linear multi-agent systems with multiple leaders of bounded inputs using distributed continuous controllers," International Journal of Robust and Nonlinear Control, vol. 25, no. 13, pp. 2101-2121, 2015.

[16] J. Hu, A. E. Turgut, B. Lennox, and F. Arvin, "Robust formation coordination of robot swarms with nonlinear dynamics and unknown disturbances: Design and experiments," IEEE Transactions on Circuits and Systems II: Express Briefs, (in press), 2021.

[17] H. Zhang, F. Lewis, and A. Das, "Optimal design for synchronization of cooperative systems: state feedback, observer and output feedback," IEEE Transactions on Automatic Control, vol. 56, no. 8, pp. 1948-1952, 2011.

[18] F. Arvin, J. Espinosa, B. Bird, A. West, S. Watson, and B. Lennox, "Mona: an affordable open-source mobile robot for education and research," Journal of Intelligent \& Robotic Systems, pp. 1-15, 2018.

[19] T. Krajník, M. Nitsche, J. Faigl, P. Vaněk, M. Saska, L. Přeučil, T. Duckett, and M. Mejail, "A practical multirobot localization system," Journal of Intelligent \& Robotic Systems, vol. 76, no. 3-4, pp. 539-562, 2014. 